\title{
Radical Structures of Fuzzy Polynomial Ideals in a Ring
}

\author{
Hee Sik Kim, ${ }^{1}$ Chang Bum Kim, ${ }^{2}$ and Keum Sook So ${ }^{3}$ \\ ${ }^{1}$ Department of Mathematics, Hanyang University, Seoul 133-791, Republic of Korea \\ ${ }^{2}$ Department of Mathematics, Kookmin University, Seoul 136-702, Republic of Korea \\ ${ }^{3}$ Department of Mathematics, Hallym University, Chuncheon 200-702, Republic of Korea \\ Correspondence should be addressed to Keum Sook So; ksso@hallym.ac.kr
}

Received 13 November 2015; Accepted 28 February 2016

Academic Editor: Cengiz Çinar

Copyright (C) 2016 Hee Sik Kim et al. This is an open access article distributed under the Creative Commons Attribution License, which permits unrestricted use, distribution, and reproduction in any medium, provided the original work is properly cited.

We investigate the radical structure of a fuzzy polynomial ideal induced by a fuzzy ideal of a ring and study its properties. Given a fuzzy ideal $\beta$ of $R$ and a homomorphism $f: R \rightarrow R^{\prime}$, we show that if $f_{x}$ is the induced homomorphism of $f$, that is, $f_{x}\left(\sum_{i=0}^{n} a_{i} x^{i}\right)=$ $\sum_{i=0}^{n} f\left(a_{i}\right) x^{i}$, then $f_{x}^{-1}\left[(\sqrt{\beta})_{x}\right]=\left(\sqrt{f^{-1}(\beta)}\right)_{x}$.

\section{Introduction}

Zadeh [1] introduced the notion of a fuzzy subset $A$ of a set $X$ as a function from $X$ into $[0,1]$. Rosenfeld [2] applied this concept to the theory of groupoids and groups. Liu [3] introduced and studied the notion of the fuzzy ideals of a ring. Following Liu, Mukherjee and Sen [4] defined and examined the fuzzy prime ideals of a ring. The concept of fuzzy ideals was applied to several algebras: $B N$-algebras [5], $B L$-algebras [6], semirings [7], and semigroups [8]. Ersoy et al. [9] applied the concept of intuitionistic fuzzy soft sets to rings, and Shah et al. [10] discussed intuitionistic fuzzy normal subrings over a nonassociative ring. Prajapati [11] investigated residual of ideals of an $L$-ring. Dheena and Mohanraj [12] obtained a condition for a fuzzy small right ideal to be fuzzy small prime right ideal.

The present authors [13] introduced the notion of a fuzzy polynomial ideal $\alpha_{x}$ of a polynomial ring $R[x]$ induced by a fuzzy ideal $\alpha$ of a ring $R$ and obtained an isomorphism theorem of a ring of fuzzy cosets of $\alpha_{x}$. It was shown that a fuzzy ideal $\alpha$ of a ring is fuzzy prime if and only if $\alpha_{x}$ is a fuzzy prime ideal of $R[x]$. Moreover, we showed that if $\alpha_{x}$ is a fuzzy maximal ideal of $R[x]$, then $\alpha$ is a fuzzy maximal ideal of $R$.

In this paper we investigate the radical structure of a fuzzy polynomial ideal induced by a fuzzy ideal of a ring and study their properties.

\section{Preliminaries}

In this section, we review some definitions which will be used in the later section. Throughout this paper unless stated otherwise all rings are commutative rings with identity.

Definition 1 (see [3]). A fuzzy ideal of a ring $R$ is a function $\alpha: R \rightarrow[0,1]$ satisfying the following axioms:

(1) $\alpha(x+y) \geq \min \{\alpha(x), \alpha(y)\}$.

(2) $\alpha(x y) \geq \max \{\alpha(x), \alpha(y)\}$.

(3) $\alpha(-x)=\alpha(x)$

for any $x, y \in R$.

Definition 2 (see [2]). Let $f: R \rightarrow S$ be a homomorphism of rings and let $\beta$ be a fuzzy subset of $S$. We define a fuzzy subset $f^{-1} \beta$ of $R$ by $f^{-1} \beta(x):=\beta(f(x))$ for all $x \in R$.

Definition 3 (see [2]). Let $f: R \rightarrow S$ be a homomorphism of rings and let $\alpha$ be a fuzzy subset of $R$. We define a fuzzy subset $f(\alpha)$ of $S$ by

$$
\begin{aligned}
f(\alpha)(y) & \\
: & = \begin{cases}\sup \{\alpha(t) \mid t \in R, f(t)=y\} & \text { if } f^{-1}(y) \neq \emptyset, \\
0 & \text { if } f^{-1}(y)=\emptyset .\end{cases}
\end{aligned}
$$


Definition 4 (see [2]). Let $R$ and $S$ be any sets and let $f: R \rightarrow$ $S$ be a function. A fuzzy subset $\alpha$ of $R$ is called an $f$-invariant if $f(x)=f(y)$ implies $\alpha(x)=\alpha(y)$, where $x, y \in R$.

Zadeh [1] defined the following notions. The union of two fuzzy subsets $\alpha$ and $\beta$ of a set $S$, denoted by $\alpha \cup \beta$, is a fuzzy subset of $S$ defined by

$$
(\alpha \cup \beta)(x):=\max \{\alpha(x), \beta(x)\}
$$

for all $x \in S$.

The intersection of $\alpha$ and $\beta$, symbolized by $\alpha \cap \beta$, is a fuzzy subset of $S$, defined by

$$
(\alpha \cap \beta)(x):=\min \{\alpha(x), \beta(x)\}
$$

for all $x \in S$.

Theorem 5 (see [13]). Let $\alpha: R \rightarrow[0,1]$ be a fuzzy ideal of $a$ ring $R$ and let $f(x)=a_{0}+a_{1} x+a_{2} x^{2}+\cdots+a_{n} x^{n}$ be $a$ polynomial in $R[x]$. Define a fuzzy set $\alpha_{x}: R[x] \rightarrow[0,1]$ by $\alpha_{x}(f(x)):=\min _{i}\left\{\alpha\left(a_{i}\right) \mid a_{i}\right.$ 's are coefficients of $\left.f(x)\right\}$. Then $\alpha_{x}$ is a fuzzy ideal of $R[x]$.

The fuzzy ideal $\alpha_{x}$ discussed in Theorem 5 is called the fuzzy polynomial ideal [13] of $R[x]$ induced by a fuzzy ideal $\alpha$.

Theorem 6 (see [13]). Let $\alpha: R \rightarrow[0,1]$ be a fuzzy ideal of a ring $R$. Then $\alpha$ is a fuzzy prime ideal of $R$ if and only if $\alpha_{x}$ is a fuzzy prime ideal of $R[x]$.

Notation 1. Let $\alpha: R \rightarrow[0,1]$ be a fuzzy subset of a set $R$. We denote a level set $\alpha_{*}$ by $\alpha_{*}:=\{a \in R \mid \alpha(a)=\alpha(0)\}$, and we know that $\alpha(0) \geqq \alpha(x)$ for all $x \in R$. The set of all polynomials $f(x)=\sum_{i=0}^{m} a_{i} x^{i} \in R[x]$ whose $\alpha$ 's values $\alpha\left(a_{i}\right)$ are equal to $\alpha(0)$ for all $i=0,1, \ldots, n$, is denoted by $\alpha_{*}[x]$.

Theorem 7 (see [13]). If $\alpha$ and $\beta$ are fuzzy ideals of a ring $R$, then

(i) $(\alpha \cap \beta)_{x}=\alpha_{x} \cap \beta_{x}$,

(ii) $\alpha_{x} \cup \beta_{x} \subseteq(\alpha \cup \beta)_{x}$.

Let $f: R \rightarrow R^{\prime}$ be a homomorphism of rings. A map $f_{x}$ : $R[x] \rightarrow R^{\prime}[x]$ defined by $f_{x}\left(a_{0}+a_{1} x+\cdots+a_{n} x^{n}\right):=f\left(a_{0}\right)+$ $f\left(a_{1}\right) x+\cdots+f\left(a_{n}\right) x^{n}$ is obviously a ring homomorphism, and we call it an induced homomorphism [13] by $f$.

Theorem 8 (see [13]). Let $f: R \rightarrow R^{\prime}$ be an epimorphism of rings and let $f_{x}$ be an induced homomorphism of $f$. If $\alpha$ is an $f$-invariant fuzzy ideal of $R$, then $(f(\alpha))_{x}=f_{x}\left(\alpha_{x}\right)$.

\section{Fuzzy Polynomial Ideals}

In this section, we study some relations between the radical of the fuzzy polynomial induced by a fuzzy ideal and the radical of a fuzzy ideal of a ring.

A fuzzy ideal $\alpha: R \rightarrow[0,1]$ of a ring $R$ is called a fuzzy prime ideal [14] of $R$ if $\alpha_{*}$ is a prime ideal of $R$. A fuzzy set $\sqrt{\alpha}: R \rightarrow[0,1]$, defined as $\sqrt{\alpha}(a):=\bigvee\left\{\alpha\left(a^{n}\right) \mid n>0\right\}$, is called a fuzzy nil radical [15] of $\alpha$.
Theorem 9 (see [15]). If $\alpha: R \rightarrow[0,1]$ is a fuzzy ideal of $R$, then the fuzzy set $\sqrt{\alpha}$ is a fuzzy ideal of $R$.

Lemma 10 (see [15]). If $\alpha$ and $\beta$ are fuzzy ideals of $R$, then $\sqrt{\alpha \cap \beta}=\sqrt{\alpha} \cap \sqrt{\beta}$.

Lemma 11. If $\alpha$ and $\beta$ are fuzzy ideals of $R$, then $\sqrt{\alpha \cup \beta}=$ $\sqrt{\alpha} \cup \sqrt{\beta}$.

Proof. If $a$ is an element of $R$, then

$$
\begin{aligned}
& \sqrt{\alpha \cup \beta}(a)=\bigvee\left\{(\alpha \cup \beta)\left(a^{n}\right) \mid n>0\right\} \\
& =\bigvee\left\{\max \left\{\alpha\left(a^{n}\right), \beta\left(a^{n}\right)\right\} \mid n>0\right\} \\
& =\max \left\{\bigvee\left\{\alpha\left(a^{n}\right) \mid n>0\right\}, \bigvee\left\{\beta\left(a^{n}\right) \mid n>0\right\}\right\} \\
& =\max \{\sqrt{\alpha}(a), \sqrt{\beta}(a)\}=(\sqrt{\alpha} \cup \sqrt{\beta})(a) .
\end{aligned}
$$

This proves that $\sqrt{\alpha \cup \beta}=\sqrt{\alpha} \cup \sqrt{\beta}$.

Since $\alpha_{x}$ is a fuzzy ideal of a polynomial ring $R[x]$ by Theorem 5 , the fuzzy set $\sqrt{\alpha_{x}}: R[x] \rightarrow[0,1]$ is the fuzzy nil radical of $\alpha_{x}$. The following theorem gives that the two fuzzy nil radicals have the same value.

Theorem 12. If $\alpha: R \rightarrow[0,1]$ is a fuzzy ideal of $R$, then

$$
\left(\sqrt{\alpha_{x}}\right)_{x}=(\sqrt{\alpha})_{x}
$$

Proof. Let $f(x):=\sum_{i=0}^{m} \in R[x]$ be any element of $R[x]$. Then, by Theorem 5 , we have $\alpha_{x}\left(a_{j}^{n}\right)=\alpha_{x}\left(a_{j}^{n}+0 x+0 x^{2}+\cdots+0 x^{m}\right)=$ $\min \left\{\alpha\left(a_{j}^{n}\right), \alpha(0), \ldots, \alpha(0)\right\}=\alpha\left(a_{j}^{n}\right)$. Since $\sqrt{\alpha_{x}}$ is a fuzzy ideal of $R[x]$, we obtain

$$
\begin{aligned}
\left(\sqrt{\alpha_{x}}\right)_{x}(f(x)) & =\min _{i=0}^{m}\left\{\sqrt{\alpha_{x}}\left(a_{i}\right)\right\} \\
& =\min _{i=0}^{m}\left\{\bigvee\left\{\alpha_{x}\left(a_{i}{ }^{n}\right) \mid n>0\right\}\right\} \\
& =\min _{i=0}^{m}\left\{\bigvee\left\{\alpha\left(a_{i}^{n}\right) \mid n>0\right\}\right\} \\
& =\min _{i=0}\left\{\sqrt{\alpha}\left(a_{i}\right)\right\}=(\sqrt{\alpha})_{x}(f(x)) .
\end{aligned}
$$

This proves that $(\sqrt{\alpha})_{x}=\left(\sqrt{\alpha_{x}}\right)_{x}$.

Theorem 13. If $\alpha$ and $\beta$ are fuzzy ideals of $R$, then

$$
(\sqrt{\alpha \cap \beta})_{x}=(\sqrt{\alpha})_{x} \cap(\sqrt{\beta})_{x} .
$$


Proof. If $\alpha$ and $\beta$ are fuzzy ideals of $R$, then $\alpha_{x}$ and $\beta_{x}$ are fuzzy ideals of $R[x]$ by Theorem 5 . It follows from Theorems 12 and 7 (i) and Lemma 10 that

$$
\begin{aligned}
(\sqrt{\alpha \cap \beta})_{x} & =\left(\sqrt{(\alpha \cap \beta)_{x}}\right)_{x} \quad[\text { Theorem 12] } \\
& =\left(\sqrt{\alpha_{x} \cap \beta_{x}}\right)_{x}[\text { Theorem 7 (i)] } \\
& =\left(\sqrt{\alpha_{x}} \cap \sqrt{\beta_{x}}\right)_{x}[\text { Lemma 10] } \\
& =\left(\sqrt{\alpha_{x}}\right)_{x} \cap\left(\sqrt{\beta_{x}}\right)_{x} \quad[\text { Theorem } 7(\mathrm{i})] \\
& =(\sqrt{\alpha})_{x} \cap(\sqrt{\beta})_{x} \quad[\text { Theorem 12] }
\end{aligned}
$$

proving the theorem.

Theorem 14. If $\alpha$ and $\beta$ are fuzzy ideals of $R$, then

$$
(\sqrt{\alpha})_{x} \cup(\sqrt{\beta})_{x} \subseteq(\sqrt{\alpha \cup \beta})_{x} .
$$

Proof. Since $\alpha_{x}$ and $\beta_{x}$ are fuzzy ideals of $R[x]$ by Theorem 5 , we obtain

$$
(\sqrt{\alpha})_{x} \cup(\sqrt{\beta})_{x}=\left(\sqrt{\alpha_{x}}\right)_{x} \cup\left(\sqrt{\beta_{x}}\right)_{x}
$$

[Theorem 12]

$$
\subseteq\left(\sqrt{\alpha_{x}} \cup \sqrt{\beta_{x}}\right)_{x}
$$$$
\text { [Theorem } 7 \text { (ii)] }
$$$$
=\left(\sqrt{\alpha_{x} \cup \beta_{x}}\right)_{x} \quad \text { [Lemma 11] }
$$$$
\subseteq\left(\sqrt{(\alpha \cup \beta)_{x}}\right)_{x} \quad[\text { Theorem } 7 \text { (ii) }]
$$$$
=(\sqrt{\alpha \cup \beta})_{x} \quad[\text { Theorem 12] } .
$$

This proves that $(\sqrt{\alpha})_{x} \cup(\sqrt{\beta})_{x} \subseteq(\sqrt{\alpha \cup \beta})_{x}$.

Theorem 15. Let $\beta$ be a fuzzy ideal of $R$ and let $f: R \rightarrow R^{\prime}$ be a homomorphism of rings. If $f_{x}$ is the induced homomorphism of $f$, that is, $f_{x}\left(\sum_{i=0}^{n} a_{i} x^{i}\right)=\sum_{i=0}^{n} f\left(a_{i}\right) x^{i}$, then

$$
f_{x}^{-1}\left[(\sqrt{\beta})_{x}\right]=\left(\sqrt{f^{-1}(\beta)}\right)_{x} .
$$

Proof. Given a polynomial $g(x)=b_{0}+b_{1} x+\cdots+b_{m} x^{m} \in R[x]$, we have

$$
\begin{aligned}
& \left(\sqrt{f^{-1}(\beta)}\right)_{x}(g(x))=\min \left\{\sqrt{f^{-1} \beta}\left(b_{0}\right),\right. \\
& \left.\sqrt{f^{-1} \beta}\left(b_{1}\right), \ldots, \sqrt{f^{-1} \beta}\left(b_{m}\right)\right\} \\
& =\min \left\{\bigvee\left\{f^{-1} \beta\left(b_{0}{ }^{n}\right) \mid n>0\right\},\right. \\
& \bigvee\left\{f^{-1} \beta\left(b_{1}{ }^{n}\right) \mid n>0\right\}, \ldots, \\
& \left.\bigvee\left\{f^{-1} \beta\left(b_{m}{ }^{n}\right) \mid n>0\right\}\right\} \\
& =\min \left\{\bigvee\left\{\beta\left(f\left(b_{0}\right)^{n}\right) \mid n>0\right\},\right. \\
& \bigvee\left\{\beta\left(f\left(b_{1}\right)^{n}\right) \mid n>0\right\}, \ldots, \\
& \left.\bigvee\left\{\beta\left(f\left(b_{m}\right)^{n}\right) \mid n>0\right\}\right\}=\min \left\{\sqrt{\beta}\left(f\left(b_{0}\right)\right),\right. \\
& \left.\sqrt{\beta}\left(f\left(b_{1}\right)\right), \ldots, \sqrt{\beta}\left(f\left(b_{m}\right)\right)\right\}=(\sqrt{\beta})_{x} \\
& \cdot\left(f_{x}(g(x))\right)=f_{x}{ }^{-1}\left[(\sqrt{\beta})_{x}\right](g(x)) .
\end{aligned}
$$

This proves that $f_{x}^{-1}\left[(\sqrt{\beta})_{x}\right]=\left(\sqrt{f^{-1}(\beta)}\right)_{x}$.

Proposition 16 (see [15]). Let $f: R \rightarrow R^{\prime}$ be a ring epimorphism from $R$ onto $R^{\prime}$, and let $\alpha: R \rightarrow[0,1]$ be a fuzzy ideal of $R$. If $\alpha$ is constant on $\operatorname{Ker} f$, then $f(\sqrt{\alpha})=\sqrt{f(\alpha)}$.

Theorem 17. Let $f: R \rightarrow R^{\prime}$ be a homomorphism of rings and let $f_{x}$ be the induced homomorphism of $f$. If a fuzzy ideal $\alpha$ of $R$ is constant on $\operatorname{Ker} f$, then the fuzzy polynomial ideal $\alpha_{x}$ is constant on $\operatorname{Ker} f_{x}$.

Proof. Let $\alpha(a)=k_{0}$ for all $a \in \operatorname{Ker} f$ and let $g(x)=b_{0}+b_{1} x+$ $\cdots+b_{m} x^{m}$ be any element of $\operatorname{Ker} f_{x}$. Then $0=f_{x}(g(x))=$ $f\left(b_{0}\right)+f\left(b_{1}\right) x+\cdots+f\left(b_{m}\right) x^{m}$. It follows that $f\left(b_{i}\right)=0$ for all $i=0,1, \ldots, m$. Hence $b_{i} \in \operatorname{Ker} f$ for all $i=0,1, \ldots, m$; that is, $\alpha\left(b_{i}\right)=k_{0}$ for all $i=0,1, \ldots, m$. This shows that $\alpha_{x}(g(x))=$ $\min \left\{\alpha\left(b_{0}\right), \alpha\left(b_{1}\right), \ldots, \alpha\left(b_{m}\right)\right\}=k_{0}$, proving the theorem.

Corollary 18. Let $f: R \rightarrow R^{\prime}$ be an epimorphism of rings and let $f_{x}$ be the induced homomorphism of $f$. If an $f$-invariant fuzzy ideal $\alpha$ of $R$ is constant on $\operatorname{Ker} f$, then

$$
f_{x}\left(\sqrt{\alpha_{x}}\right)=\sqrt{(f(\alpha))_{x}} .
$$

Proof. It follows from Proposition 16 and Theorem 8 that $f_{x}\left(\sqrt{\alpha_{x}}\right)=\sqrt{f_{x}\left(\alpha_{x}\right)}=\sqrt{(f(\alpha))_{x}}$.

Theorem 19. Let $\alpha$ be a fuzzy ideal of $R$ and let $\alpha_{x}$ be its fuzzy polynomial ideal of $R[x]$. If $\beta$ is a fuzzy prime ideal of $R[x]$ such that $\alpha_{x} \leqq \beta$, then there exists a fuzzy prime ideal $\alpha_{0}$ of $R$ such that $\left(\alpha_{0}\right)_{*}=\beta_{*} \cap R$ and $\alpha \leqq \alpha_{0}$.

Proof. Since $\beta$ is a fuzzy prime ideal of $R[x], \beta_{*}$ is a prime ideal of $R[x]$. If we define $\gamma:=\beta_{*} \cap R$, then it is easy to show 
that $\gamma$ is a prime ideal of $R$. Define a fuzzy subset $\alpha_{0}: R \rightarrow$ $[0,1]$ by

$$
\alpha_{0}(a)= \begin{cases}\beta(0) & \text { if } a \in \gamma, \\ \beta(a) & \text { if } a \notin \gamma .\end{cases}
$$

Then, by routine calculations, we show that $\alpha_{0}$ is a fuzzy ideal of $R$ satisfying $\left(\alpha_{0}\right)_{*}=\gamma$. We claim that $\alpha \subseteq \alpha_{0}$. Given $a \in R$, if $a \in \gamma$, then $\alpha(a)=\alpha_{x}(a) \leq \beta(a) \leq \beta(0)=\alpha_{0}(a)$. If $a \notin \gamma$, then $\alpha(a)=\alpha_{x}(a) \leq \beta(a)=\alpha_{0}(a)$. Since $\gamma$ is a prime ideal of $R,\left(\alpha_{0}\right)_{*}$ is a prime ideal of $R$. This shows that $\alpha_{0}$ is a fuzzy prime ideal of $R$, proving the theorem.

Definition 20 (see [15]). Let $\alpha: R \rightarrow[0,1]$ be a fuzzy ideal of $R$. The fuzzy ideal $r(\alpha)$ defined by

$$
r(\alpha):=\bigcap\{\beta \mid \alpha \subseteq \beta, \beta: \text { a fuzzy prime ideal of } R\}
$$

is called the prime fuzzy radical of $\alpha$.

Theorem 21. Let $\alpha$ be a fuzzy ideal of $R$ and let $\alpha_{x}$ be its fuzzy polynomial ideal of $R[x]$. Then

$$
r\left(\alpha_{x}\right) \subseteq(r(\alpha))_{x} .
$$

Proof. By Theorem $6, \beta_{i}$ is a fuzzy prime ideal of $R$ with $\alpha \subseteq \beta_{i}$ if and only if $\left(\beta_{i}\right)_{x}$ is a fuzzy prime ideal of $R[x]$ with $\alpha_{x} \subseteq$ $\left(\beta_{i}\right)_{x}$. It follows from Theorem 7(i) that

$$
\begin{aligned}
& (r(\alpha))_{x}=\left(\bigcap \left\{\beta_{i} \mid \alpha\right.\right. \\
& \left.\left.\quad \subseteq \beta_{i}, \beta_{i} \text { is a fuzzy prime ideal of } R\right\}\right)_{x} \\
& \quad=\left(\bigcap \left\{\left(\beta_{i}\right)_{x} \mid \alpha\right.\right. \\
& \left.\left.\quad \subseteq \beta_{i}, \beta_{i} \text { is a fuzzy prime ideal of } R\right\}\right)=\bigcap\left\{\left(\beta_{i}\right)_{x} \mid\right. \\
& \left.\alpha_{x} \subseteq\left(\beta_{i}\right)_{x},\left(\beta_{i}\right)_{x} \text { is a fuzzy prime ideal of } R[x]\right\} \\
& \supseteq \bigcap\left\{\gamma_{i} \mid \alpha_{x} \subseteq \gamma_{i},\right. \\
& \left.\gamma_{i} \text { is a fuzzy prime ideal of } R[x]\right\}=r\left(\alpha_{x}\right),
\end{aligned}
$$

proving the theorem.

Notation 2. Let $\alpha$ be a fuzzy ideal of $R$ and let $\alpha_{x}$ be its fuzzy polynomial ideal of $R[x]$. We denote $\operatorname{FPI}(\alpha)$ by

$$
\text { FPI }(\alpha):=\{\beta \mid \alpha \subseteq \beta, \beta \text { is a fuzzy prime ideal of } R\}
$$

and $\operatorname{FPI}\left(\alpha_{x}\right)$ by

$$
\begin{aligned}
& \operatorname{FPI}\left(\alpha_{x}\right) \\
& \quad:=\left\{\gamma \mid \alpha_{x} \subseteq \gamma, \gamma \text { is a fuzzy prime ideal of } R[x]\right\} .
\end{aligned}
$$

Theorem 22. Let $\alpha$ be a fuzzy ideal of $R$ and let $\alpha_{x}$ be its fuzzy polynomial ideal of $R[x]$. Then a map $\phi: \operatorname{FPI}(\alpha) \rightarrow \operatorname{FPI}\left(\alpha_{x}\right)$ defined by $\phi(\beta):=\beta_{x}$ is one-one.

Proof. If $\beta, \gamma \in \operatorname{FPI}(\alpha)$ such that $\phi(\beta)=\phi(\gamma)$, then $\beta_{x}=\gamma_{x}$. It follows that $\beta_{x}(a)=\gamma_{x}(a)$ for all $a \in R$, and hence $\beta(a)=\gamma(a)$ for all $a \in R$, proving that $\beta=\gamma$. Hence $\phi$ is one-one.
Corollary 23. Let $\alpha$ be a fuzzy ideal of $R$ and let $\alpha_{x}$ be its fuzzy polynomial ideal of $R[x]$. If the map $\phi$ defined in Theorem 22 is an onto map, then

$$
(r(\alpha))_{x}=r\left(\alpha_{x}\right) .
$$

Proof. If $\beta$ is any element of $\operatorname{FPI}\left(\alpha_{x}\right)$, then there exists $\gamma \in$ $\operatorname{FPI}(\alpha)$ such that $\gamma_{x}=\phi(\gamma)=\beta$ with $\alpha \subseteq \gamma$. Thus $(r(\alpha))_{x}=$ $r\left(\alpha_{x}\right)$. This shows that the reverse inclusion in Theorem 21 holds.

Example 24. Let $\mathbf{Z}$ be set of all integers. Let

$$
\alpha(x):= \begin{cases}1 & \text { if } x \in 2 \mathbf{Z} \\ 0 & \text { if } x \notin 2 Z\end{cases}
$$

Then $\alpha$ is a fuzzy prime ideal of $\mathbf{Z}$, since $\alpha_{*}=2 \mathbf{Z}$ is a prime ideal of $\mathbf{Z}$, and its induced polynomial ideal $\alpha_{x}$ is

$$
\alpha_{x}(f(x)):= \begin{cases}1 & \text { if } f(x) \in 2 \mathbf{Z}[x], \\ 0 & \text { if } f(x) \notin 2 \mathbf{Z}[x] .\end{cases}
$$

By Theorem 6 , the fuzzy polynomial ideal $\alpha_{x}$ induced by $\alpha$ is a fuzzy prime ideal of $\mathbf{Z}[x]$. Hence $(r(\alpha))_{x}=\alpha_{x}=r\left(\alpha_{x}\right)$.

\section{Competing Interests}

The authors declare that they have no competing interests.

\section{Acknowledgments}

This research was supported by Hallym University Research Fund, 2015 (HRF-201503-016).

\section{References}

[1] L. A. Zadeh, "Fuzzy sets," Information and Control, vol. 8, no. 3, pp. 338-353, 1965.

[2] A. Rosenfeld, "Fuzzy groups," Journal of Mathematical Analysis and Applications, vol. 35, pp. 512-517, 1971.

[3] W. J. Liu, "Fuzzy invariant subgroups and fuzzy ideals," Fuzzy Sets and Systems, vol. 8, no. 2, pp. 133-139, 1982.

[4] T. K. Mukherjee and M. K. Sen, "On fuzzy ideals of a ring I," Fuzzy Sets and Systems, vol. 21, no. 1, pp. 99-104, 1987.

[5] G. Dymek and A. Walendziak, "(Fuzzy) ideals of BN-algebras," The Scientific World Journal, vol. 2015, Article ID 925040, 9 pages, 2015.

[6] B. L. Meng and X. L. Xin, "On fuzzy ideals of BL-algebras," The Scientific World Journal, vol. 2014, Article ID 757382, 12 pages, 2014.

[7] H. V. Kumbhojkar, "Proper fuzzification of prime ideals of a hemiring," Advances in Fuzzy Systems, vol. 2012, Article ID 801650, 8 pages, 2012.

[8] M. Khan, F. Feng, and M. N. A. Khan, "On minimal fuzzy ideals of semigroups," Journal of Mathematics, vol. 2013, Article ID 475190, 5 pages, 2013.

[9] B. A. Ersoy, S. Onar, K. Hila, and B. Davvaz, "Some properties of intuitionistic fuzzy soft rings," Journal of Mathematics, vol. 2013, Article ID 650480, 8 pages, 2013. 
[10] T. Shah, N. Kausar, and I. Rehman, "Intuitionistic fuzzy normal subring over a non-associative ring," Analele Universitatii 'Ovidius' Constanta, vol. 20, no. 1, pp. 369-386, 2012.

[11] A. S. Prajapati, "Residual of ideals of an L-ring," Iranian Journal of Fuzzy Systems, vol. 4, no. 2, pp. 69-82, 2007.

[12] P. Dheena and G. Mohanraj, "Fuzzy small right ideal of rings," Journal of Hyperstructures, vol. 2, pp. 8-17, 2013.

[13] C. B. Kim, H. S. Kim, and K. S. So, "On the fuzzy polynomial ideals," Journal of Intelligent and Fuzzy Systems, vol. 27, no. 1, pp. 487-494, 2014.

[14] H. V. Kumbhojkar and M. S. Bapat, "Not-so-fuzzy fuzzy ideals," Fuzzy Sets and Systems, vol. 37, no. 2, pp. 237-243, 1990.

[15] H. V. Kumbhojkar and M. S. Bapat, "On prime and primary fuzzy ideals and their radicals," Fuzzy Sets and Systems, vol. 53, no. 2, pp. 203-216, 1993. 


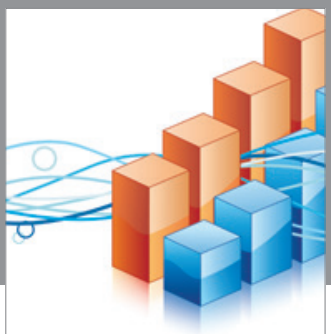

Advances in

Operations Research

vatem alat4

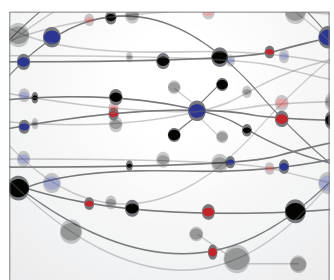

\section{The Scientific} World Journal
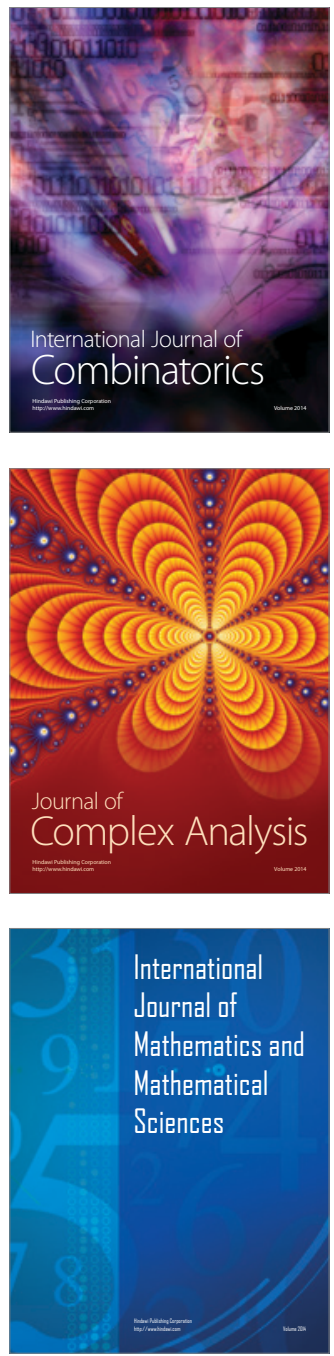
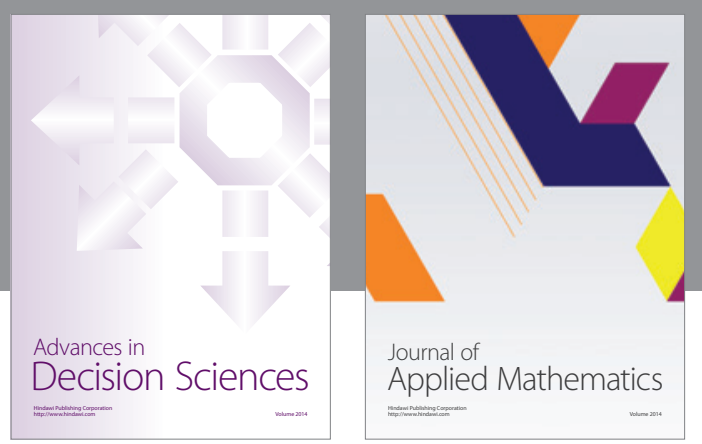

Algebra

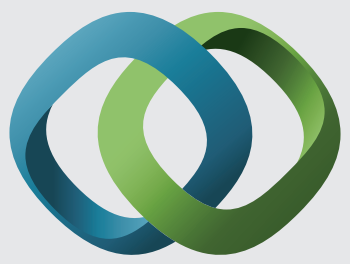

\section{Hindawi}

Submit your manuscripts at

http://www.hindawi.com
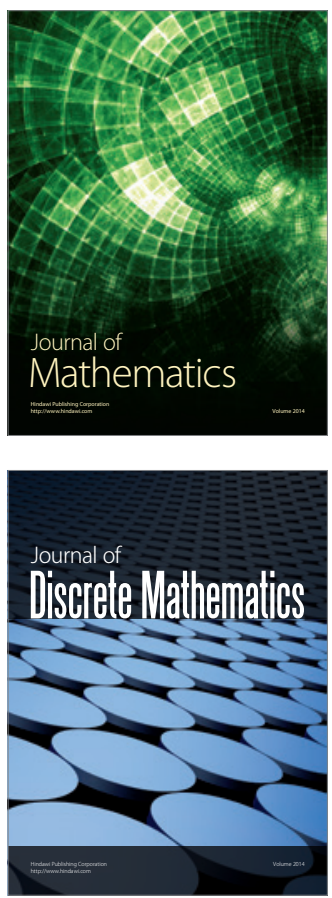

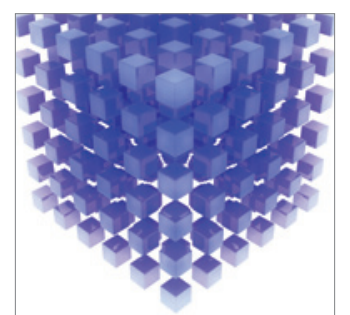

Mathematical Problems in Engineering
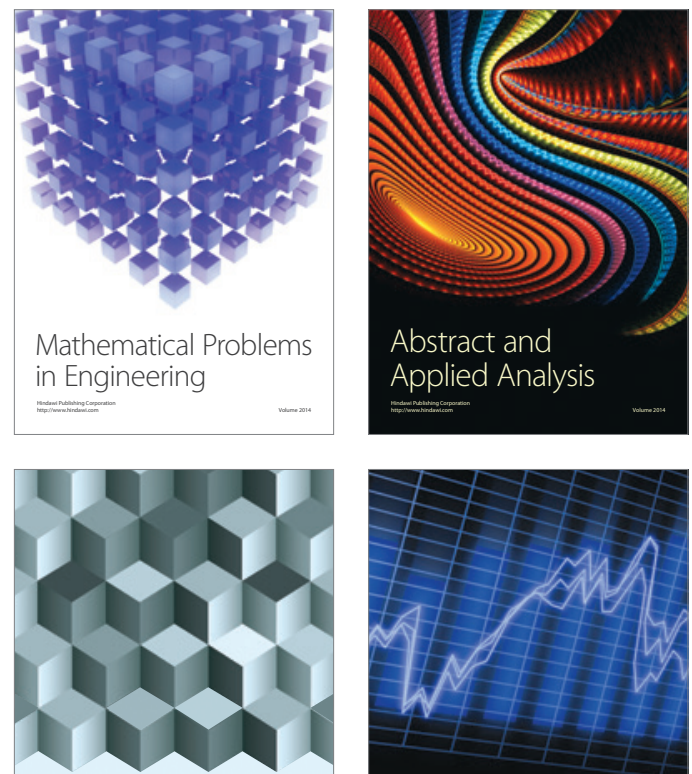

Journal of

Function Spaces

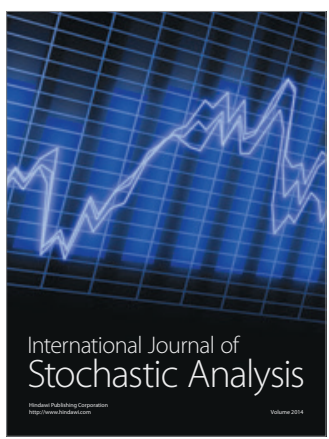

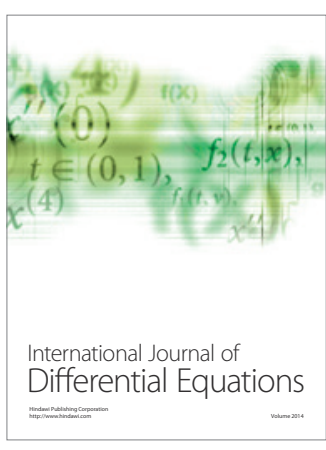
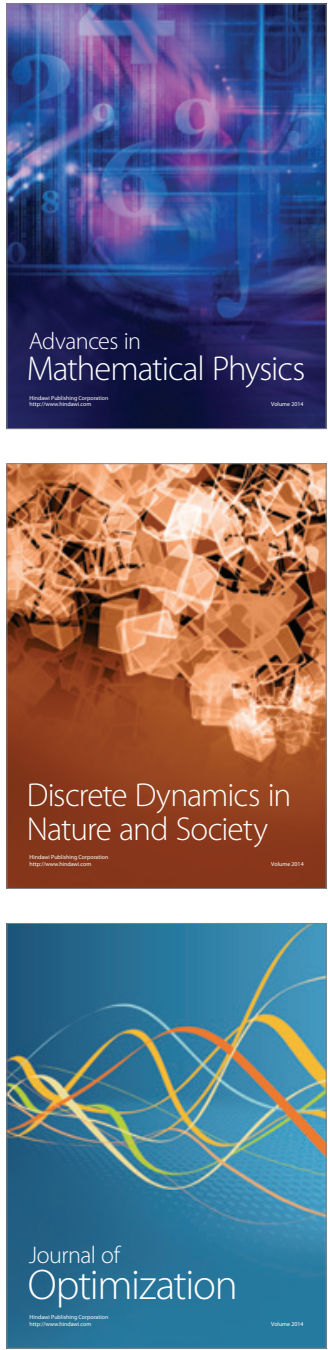